\title{
First steps in the development of a piston sensor for large aperture space telescopes
}

\author{
Géraldine Guerri $^{a, b}$, Stéphane Roose ${ }^{a}$, Yvan $\operatorname{Stockman}^{a}$, Alexandra Mazzoli ${ }^{a}$, Jean Surdej ${ }^{b}$ and Jean- \\ Marc Defise $^{a}$ \\ ${ }^{a}$ Centre Spatial de Liège (CSL), Avenue Pré-Aily, B-4031 Angleur, Belgium; \\ ${ }^{b}$ Institut d'Astrophysique et de Géophysique, Université de Liège, Allée du 6 Août, B-4000 Liège, \\ Belgium
}

\begin{abstract}
Nowadays spaceborne missions for astronomy or Earth imaging need high resolution observation which implies the development of large aperture telescopes. This can be achieved by multi-aperture telescopes or large segmented telescopes. One of the major issues is the phasing of the sub-apertures or the segments of such telescopes. A cophasing sensor is therefore mandatory to achieve the ultimate resolution of these telescopes.

In this framework, Liège Space Center (CSL) concern is the development of a compact cophasing sensor to phase new large lightweight segmented mirrors for future space telescopes. The sensor concept has its origins in new phase retrieval algorithms which have been recently developed.

In this paper, we outline the concept and the experimental validation results of our piston sensor breadboard which is currently under development in our laboratory. Finally, future prospects and further developments of our experiment are presented.
\end{abstract}

Keywords: Phase Retrieval, Cophasing, Segmented Telescopes

\section{INTRODUCTION}

Multiple Aperture Optical Telescopes (MAOT) or Large Segmented Telescopes (LST) are nowadays considered for high-resolution spaceborne missions such as Earth imaging or astronomy.

To perform real-time correction of mechanical disturbance in these high-resolution telescopes deployed after launch, a critical component is the wavefront sensor and particularly the cophasing sensor, whose goal is to measure the relative positioning (piston, tip and tilt errors) of MAOT subapertures or LST segments, or even the high-order aberrations on each segment.

\subsection{Brief state of the art of the existing cophasing sensor concepts}

Several types of wavefront sensors have been studied and investigated in the astronomical community. We can list these sensors in 2 main categories:

1. Pupil plane detection sensors: they split the pupil into subapertures in some way, then use intensity in each subaperture to deduce the phase of the wavefront. There are 2 sub-categories :

- Slope sensing: Shack-Hartmann ${ }^{13}$, pyramid sensing ${ }^{9}$

- Curvature sensing ${ }^{11}$

The Shack-Hartmann wavefront sensor is a simple and elegant means for measuring the shape of a wavefront. This technique has become widespread throughout the world and has found a wide variety of applications. The other sensors are younger and we will know more about their effective performances within the results of the Active Phasing Experiment (APE) ${ }^{6,17}$ which aims at studying new phasing technologies and at validating wavefront control concepts for Extremely Large Telescopes. The four different phasing sensors (curvature ${ }^{11}$, pyramid ${ }^{9}$, Shack-Hartmann ${ }^{13}$ sensors and $^{2}$ phase contrast interferometer ${ }^{16}$ ) have been tested in parallel to compare them under the same conditions (Gonte et al., $2008^{5}$ ) and have been tested on sky (Gonte et al., 20097). The promising results that have been obtained foresee that these concepts will be suitable for future large ground-based telescopes. 
2. Focal plane detection sensors : wavefront properties are deduced from whole-aperture intensity measurements made at or near the focal plane. There are 2 sub-categories :

- Dispersed fringe sensing ${ }^{14}$, Phase-shifting interferometer ${ }^{15}$

- Phase diversity ${ }^{3}$, phase retrieval ${ }^{1,4}$

Dispersed fringe sensors and phase-shifting interferometer are well-known and commonly used ; they can sometimes be bulky and are preferable for coarse phasing. Several phase retrieval/diversity algorithms have been developed both for point-source or extended objects : they are easy to implement ; however they are iterative and consequently time consuming. But actually new real-time phase retrieval $^{2} /$ diversity $^{8}$ algorithms have been proposed and studied to be used in closed-loop in the case of small phase perturbations. They can be the solution for fine phasing for the future space missions.

\subsection{Trade-off and sensor concept selection}

After this state of the art review, a trade-off was done to determine the sensor concepts that match better our need. The cophasing sensor requirements are the following:

- co-phasing of 3 to 7 sub-apertures

- separate measurement of piston and tip/tilt

- low weight and compactness

- reduced hardware complexity

- real-time correction

- linearity, high range and accuracy, robustness

Finally, the sensor concepts that have been retained after the trade-off are listed in Table 1. Note that two working regimes have to be considered for a cophasing sensor:

- Coarse phasing in open loop

- Fine phasing in closed loop when the piston, tip or tilt errors are less than $\lambda / 2$

\begin{tabular}{|l|c|c|}
\hline & PISTON sensing & TIP-TILT sensing \\
\hline $\begin{array}{l}\text { Coarse } \\
\text { phasing }\end{array}$ & $\begin{array}{c}\text { Dispersed fringe sensing } \\
\text { Fine phasing } \\
\text { (Error } \leq \lambda / \mathbf{2})\end{array}$ & $\begin{array}{c}\text { Shack-Hartmann sensor } \\
\left(\text { Shack \& Platt, } 1971^{13} \text { ) }\right.\end{array}$ \\
\hline & $\begin{array}{c}\text { (Real-time' phase retrieval } \\
\left(\text { Baron et al., 2008 }{ }^{2}\right)\end{array}$ & $\begin{array}{c}\text { Shack-Hartmann sensor }{ }^{13} \\
\text { or }\end{array}$ \\
& $\begin{array}{c}\text { 'Real-time' phase diversity } \\
(\text { Mocoeur et al., 2007 }\end{array}$ \\
\hline
\end{tabular}

Table 1. Inventory of the selected concepts for the CSL cophasing sensor.

The trade-off criteria were the best compliance with the requirements, the sensor maturity and the feasibility of a breadboard within a short term. We first decided to study the feasibility of the piston sensor in the fine phasing regime that is the most critical part of the cophasing sensor. In the remainder of this article, we therefore focus on the fine piston sensing by the real-time phase retrieval method ${ }^{2}$.

\section{PISTON SENSOR NUMERICAL VALIDATION}

In this section, we present the numerical validation of the real-time phase algorithm ${ }^{2}$ that we retained for the fine piston estimation. With this technique, the piston errors are retrieved from one focal-plane intensity measurement. Provided that the piston aberrations are small enough (ie small compared to $2 \pi \mathrm{rad}$, for fine cophasing in closed-loop operation) and that the pupil is diluted noncentrosymetric, Baron et al. ${ }^{2}$ have developed an analytical and real-time estimator adopting a least-square approach. This algorithm is based on the computation of the Optical Transfer Function (OTF) and therefore needs only one FFT computation.

The simulations are performed with a wavelength $\lambda$ of $633 \mathrm{~nm}$. The object is a point source and the chosen pupil is shown on the left of Fig. 1 : the first numerical validation is performed for a 3 sub-apertures telescope. The dilution factor, defined as the shortest baseline divided by the sub-aperture diameter, is equal to 1.72 . Images with $256 \times 256$ pixels are simulated. Fig. 1 shows the effect on the PSF and the OTF when a piston error is introduced on one subaperture. 


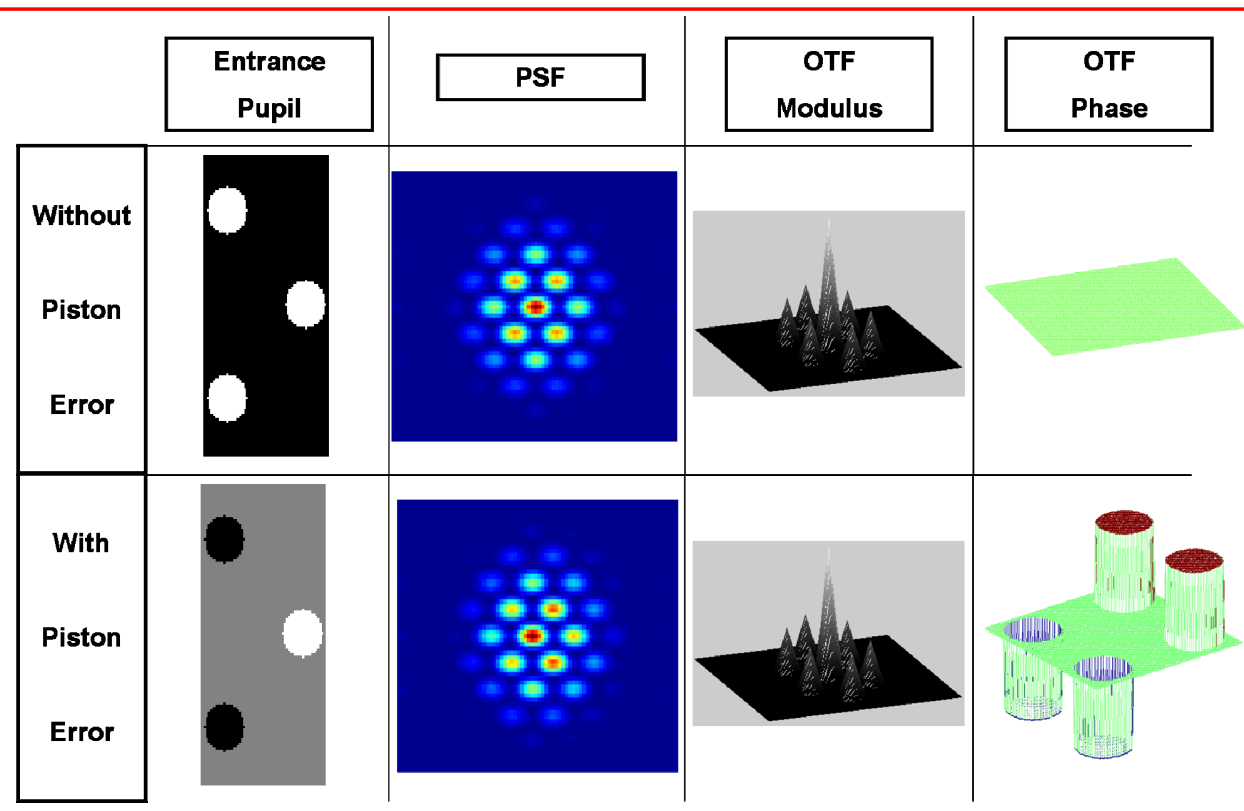

Figure 1. Effects on the PSF and the OTF due to the presence of a piston error on one of the 3 telescope sub-apertures.

The presence of a piston error induces a disruption on the OTF phase by affecting the intensity of the $2\left(\mathrm{~N}_{\mathrm{t}}-1\right)$ OTF's satellite peaks $\left(\mathrm{N}_{\mathrm{t}}\right.$ being the number of sub-apertures) : the OTF modulus is strictly unaffected, whereas a piston aberration creates a phase offset in the OTF phase.

The linearity curve, obtained from a piston ramp of $[-\lambda, \lambda]$ is presented in Fig. 2.

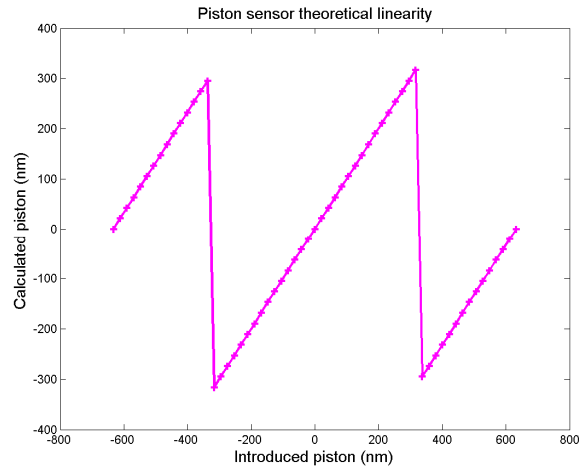

Figure 2. Response of the phase retrieval analytical estimator to a piston ramp for a 3 aperture telescope.

We can see on this figure that the algorithm is linear over the domain, with a wrapping of $\lambda$ when the modulus of the amplitude increases beyond $\lambda / 2$. This is a classical result for monochromatic piston estimation. On each linear part, the slope of the curve is equal to unity.

Furthermore, we tested the same algorithm for a 7 aperture telescope. The telescope configuration and the resulting PSF and OTF with and without piston error are shown on figure 3. Note that it is very important to choose a non redundant configuration to avoid the overlapping of the OTF peaks. Within this configuration, the OTF is composed of $\mathrm{N}_{\mathrm{t}}\left(\mathrm{N}_{\mathrm{t}}-1\right)$ ie 42 satellite peaks and the presence of a piston error on one sub-aperture affects the intensity of the OTF phase's 12 peaks. 


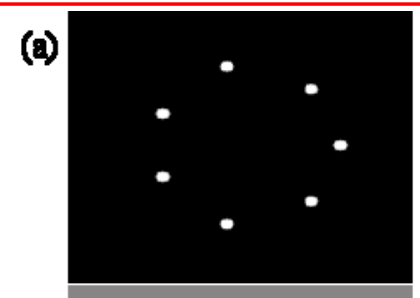

$(\theta)$

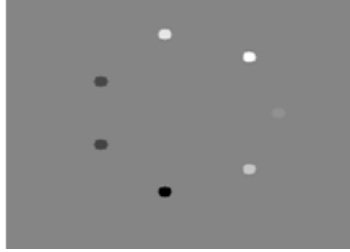

(b)

(f)

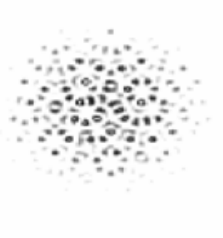

(c)

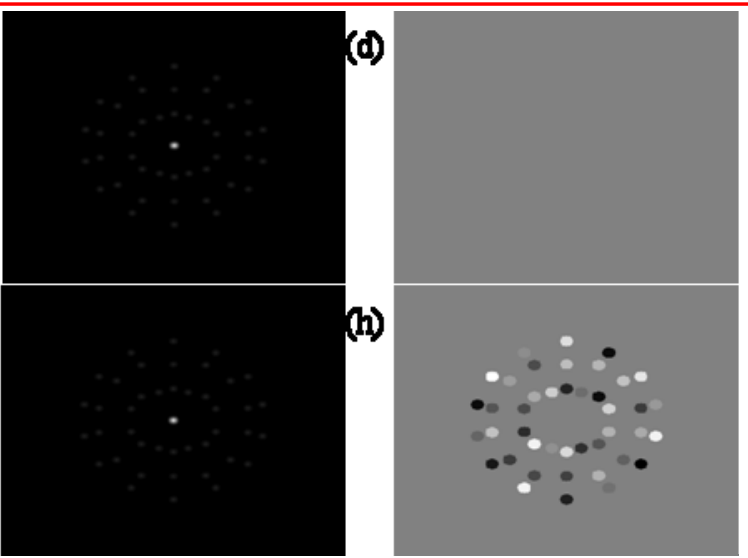

Figure 3. Effects on the PSF and the OTF due to the presence of a piston error on one of the 7 telescope sub-apertures (bottom) compared to the case without piston error (top) : (a)\&(e) entrance pupil, (b)\&(f) PSF, (c)\&(g) OTF modulus, (d)\&(h) OTF phase.

The linearity curve displayed in Fig. 4 attests that the fine cophasing of a 7 aperture telescope is also possible with this algorithm.

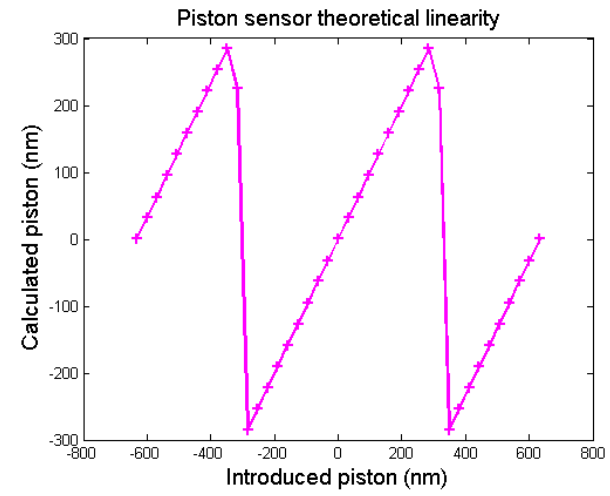

Figure 4. Response of the phase retrieval analytical estimator to a piston ramp for a 7 aperture telescope.

Finally, we conclude that these simulations comply with the requirements and allow the realization of a breadboard that will be presented in the next section.

\section{PISTON SENSOR EXPERIMENTAL VALIDATION}

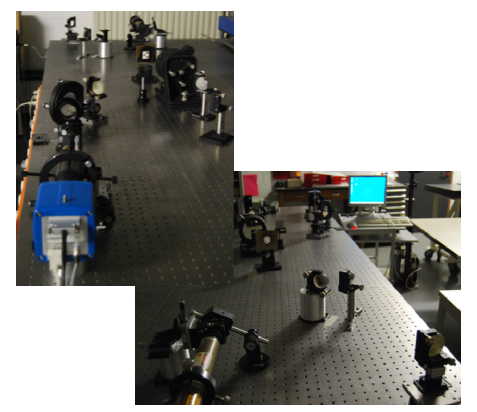

Figure 5. Pictures of the optical bench. 
In order to evaluate the feasibility and the performance of the focal-plane piston sensor using the phase retrieval analytical algorithm ${ }^{2}$, a validation breadboard has been built, using standard optical elements that are available in the laboratory. Pictures of the bench are shown in Fig. 5.

\subsection{Breadboard's principle and optical setup}

Fig. 6 shows the optical setup of our piston sensor validation breadboard. The inventory and the properties of each component of the breadboard are given in Table 2.

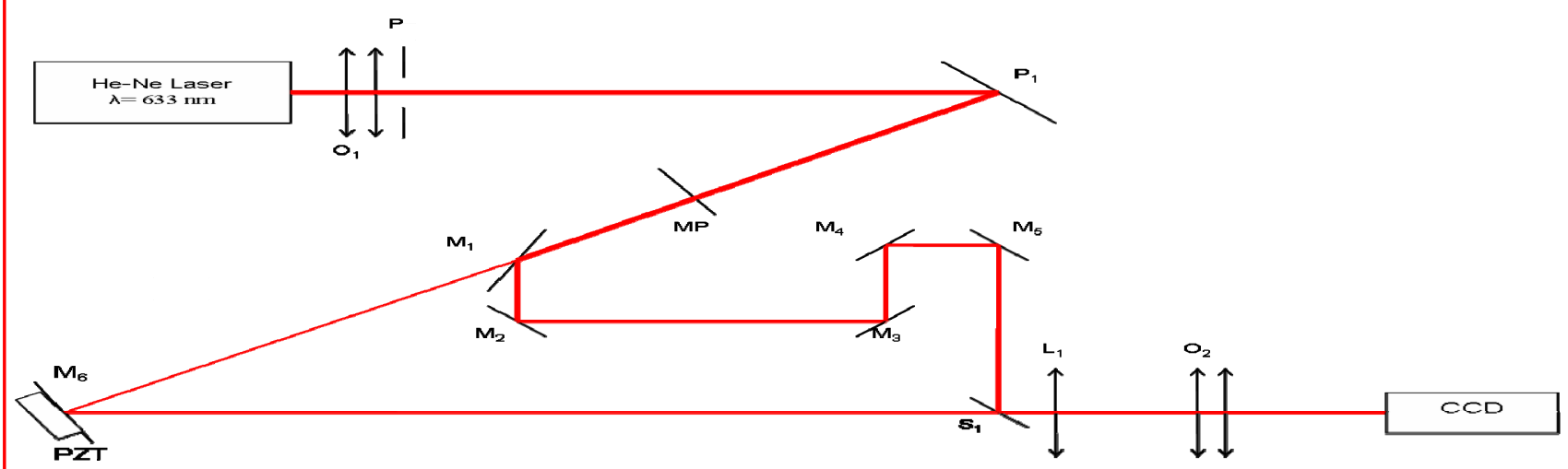

Figure 6. Optical setup of the piston sensor validation breadboard.

A collimated parallel beam is obtained at the output of the off-axis parabola $\mathrm{P}_{1}$ from a HeNe Laser $(\lambda=632.8 \mathrm{~nm})$. The pupil mask MP reproduces the configuration of a 3 sub-aperture telescope. For this experimental validation, we have selected a non-centrosymetric configuration, the 3 sub-apertures are set in an isosceles layout with a dilution factor of 1.72 .

\begin{tabular}{|c|c|}
\hline Component & Characteristics \\
\hline HeNe Laser Melles Griot & $\lambda=632.8 \mathrm{~nm}, \mathrm{P}=5 \mathrm{~mW}$, unpolarised \\
\hline Microscope objective $\mathrm{O}_{1}$ & $\Phi=5 \mu \mathrm{m}$ \\
\hline Pinhole $\mathrm{P}$ & $\mathrm{f}=+750 \mathrm{~mm}$, optical quality $\lambda / 5, \Phi=125 \mathrm{~mm}$ \\
\hline Off-axis parabola $\mathrm{P}_{1}$ & isosceles layout, 1.72 dilution factor \\
\hline Pupil mask & Zerodur, $\Phi=50.8 \mathrm{~mm}, \mathrm{R} \geq 96 \%$, optical quality $\lambda / 10$ \\
\hline Plane mirrors $\mathrm{M}_{1}, \mathrm{M}_{2}, \mathrm{M}_{3}, \mathrm{M}_{4}, \mathrm{M}_{5}$ and $\mathrm{M}_{6}$ & Piezosystem jena $\mathrm{PX} 200,200 \mu \mathrm{m}$ motion, $0.37 \mathrm{~nm}$ resolution \\
\hline Piezo actuator PZT & BK7, $\Phi=75 \mathrm{~mm}, \mathrm{R}=\mathrm{T} \approx 50 \%$ in the visible, optical quality $\lambda / 10$ \\
\hline Beamsplitter $\mathrm{S}_{1}$ & $\mathrm{f}_{1}=+300 \mathrm{~mm}, \Phi=50.8$ mm \\
\hline Imaging converging lens $\mathrm{L}_{1}($ achromat) & $\mathrm{X} 60$ \\
\hline Microscope objective $\mathrm{O}_{2}$ & $2048 \times 2048$ pixels, $14 \mu \mathrm{m}$ pixel size, 8 bits dynamics \\
\hline CCD Camera Atmel-Camelia $4 \mathrm{M}$ &
\end{tabular}

Table 2. Inventory of the CSL piston sensor validation breadboard components.

Then, the entrance pupil is divided in two paths in order to introduce a piston error on one sub-aperture :

- $\quad$ The plane mirror $\mathrm{M}_{1}$ reflects the light from only 2 sub-apertures over 3.

- The light from the isolated sub-aperture is reflected by the mirror $\mathrm{M}_{6}$, this mirror is mounted on a piezoelectric transducer in order to introduce a piston error.

- The mirrors $\mathrm{M}_{2}, \mathrm{M}_{3}, \mathrm{M}_{4}$ and $\mathrm{M}_{5}$ form a delay line to minimize the optical path difference between all the sub-apertures.

After the beam-splitter come the components of the piston sensor strictly speaking : the focal lens $\mathrm{L}_{1}$ and the microscope $\mathrm{O}_{2}$ produce a focal-plane image on the detector. It is this image that will be used to retrieve the piston error. The detector is a CCD camera whose main characteristics are : array of 2048x2048 pixels (14 $\mu \mathrm{m}$ each), 8 bits dynamics. 


\subsection{Measurement principle}

The phase retrieval algorithm needs to acquire a pupil image for the preliminary calibration and a focal image of the PSF for the measurement. As the piston error is retrieved from intensity measurement, it is necessary to control the uniformity of the incident light beam intensity and to avoid straylight. Dark image subtraction is therefore mandatory.

Generally speaking, piston sensors are very difficult to align because of the intrinsic piston, the pupil superposition and the stability of the alignment. In our case, we noticed that the measurements are mainly sensitive to the chamber vibrations, the light background and the uniformity of the pupil lighting. Measurements are made at high flux ; a neutral density of 2.0 is placed just behind the laser source and the exposure time (few tenths of ms) is set to avoid saturation of the detector. Furthermore, we noticed that the precision of the phase retrieval algorithm requires that the coordinates of the PSF center are precisely well-known with a \pm 2 pixels uncertainty.

For the image acquisition, we used the software that was provided by the manufacturer of the camera. For the data processing, we developed a graphical user interface under the MATLAB ${ }^{\text {TM }}$ environment that implements the real-time phase retrieval algorithm².

\subsection{Results of the experimental validation}

We first performed linearity tests to check the validity of the phase retrieval estimator : we applied a piston ramp on the given sub-aperture ; some of the resulting images are shown in Figure 7(a). These images are in good agreement with theoretical predictions.

(a)
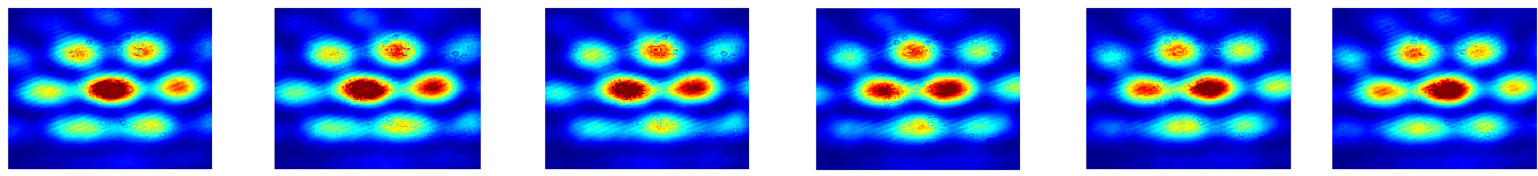

(b)

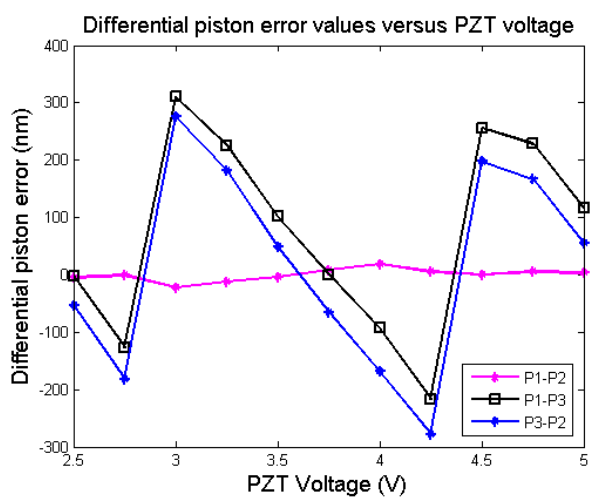

Figure 7. Experimental linearity of the piston estimator : (a) PSFs obtained during a piston scan; (b) Differential piston errors measured with the phase retrieval analytical algorithm versus the voltage applied to the piezoelectric transducer (P1, P2, P3 are respectively the sub-pupils number 1,2,3).

Figure 7(b) presents the piston estimated when a piston error is applied to the sub-aperture P3. We can see that wrapping occurs roughly near piston values equal to $\mathrm{k} \lambda / 2$ (with $\mathrm{k}$ being an integer). This is due to the intrinsic modulo $2 \pi$ dynamic range of the estimator. Between these wrappings, the linearity is very good with a slope coefficient of -0.96 . The slope coefficient is negative because increasing the voltage applied to the piezoelectric transducer induces a decrease of the optical path difference between the sub-apertures.

Besides, the algorithm computation time, obtained an a standard simple processor laptop, is below one second for data images of $256 \times 256$ pixels. This value is suitable for real-time processing and can be reduced by minimizing the image size and by developing a whole software in $\mathrm{C}++$ language that combines both the image acquisition and the data processing. We conclude that real-time correction is thus possible in closed-loop. Note that additional procedures are required to lock the system on a nearly co-phased state. 
Table 3 sums up the main characteristics of our piston sensor that have been obtained experimentally.

\begin{tabular}{|c|c|}
\hline Metrological standard & Value \\
\hline Measurement range & {$[-\lambda / 2, \lambda / 2]$} \\
\hline Linearity & $\geq 0.92($ best $: 0.96)$ \\
\hline Resolution & $\leq 20 \mathrm{~nm}$ \\
\hline Deviation around zero point (rms) & $\leq 10 \mathrm{~nm}($ best $: 6 \mathrm{~nm})$ \\
\hline Absolute error & $\pm 25 \mathrm{~nm}$ \\
\hline Repeatability & TBD \\
\hline
\end{tabular}

Table 3. Values of the metrological standards obtained experimentally for the CSL piston sensor validation breadboard.

The measured values are compliant with our expectations. We have noticed that, during a complete set of measurements, the theoretical null differential piston error value (from the two unaberrated sub-apertures) can be obtained with an absolute error less than $25 \mathrm{~nm}$ and with a rms deviation less than $10 \mathrm{~nm}$. Special care has to be taken to control the uniformity of the lighting and the optical path differences. Moreover, the resolution value is limited for the moment by the smallest voltage that can be applied to the piezoelectric transducer.

Finally, we conclude that these results are very satisfactory with our requirements and validate the feasibility study for a 3 apertures telescope. Note that the piston measurement repeatability for different source brightness was qualitatively evaluated successfully by placing increasing neutral densities behind the laser source. Improvements have to be made on the optical bench and on the camera software and the data analysis software to enhance the accuracy of the measurements and the sensor performances.

\section{CONCLUSIONS AND FUTURE PROSPECTS}

In this paper, we presented the numerical and experimental validation results of our piston sensor breadboard for the fine cophasing of a 3 sub-aperture space telescope. This piston sensor implements new 'real-time' phase retrieval algorithm ${ }^{2}$ which can retrieve small piston errors (beyond $|\lambda / 2|$ ) from a simple focal plane image and the computation on only one FFT.

We conclude that the feasibility study of this kind of sensor is validated and the real-time phase retrieval technique ${ }^{2}$ is suitable for the fine piston estimation of future large aperture space telescopes. Improvements of the breadboard are to be made to increase the overall performances, especially for reducing at maximum the image size to increase the computation time and for enhancing the optical quality for a better accuracy of the measurement.

The main future prospects are :

- Experimental tests of the fine piston sensing of 7 sub-apertures.

- Design and implementation of the tip-tilt measurement : firstly, a Shack-Hartmann sensor produced by Optocraft will be inserted in the breadboard. Then, the feasibility of the 'real-time' phase diversity ${ }^{8}$ technique will be studied and compared to the Shack-Hartmann measurements for the fine tip-tilt estimation regime.

- Cophasing of three new silicon bimorph mirrors developed at Université Libre de Bruxelles by Rodrigues et al. $\left(2009^{10}\right)$.

At longer term, the whole cophasing sensor for both piston and tip-tilt estimation will use integrated optical components to increase the compactness and the mass reduction.

\section{ACKNOWLEDGMENTS}

G. Guerri is grateful to the "Communauté Française de Belgique - Action de recherche concertée - Académie Wallonie Europe" for supporting her Post-Doctoral grant. 


\section{REFERENCES}

1. Baron, F., PhD Thesis, 2005

2. Baron, F., Mocoeur, I., Cassaing, F., Mugnier, L. M., 2008, JOSA, 25(5), 1000-1015

3. Delavaquerie, E., Meimon, S., Cassaing, F., Mocoeur, I., Fusco, T., Mugnier, L., Michau, V., 2008, Optical Complex Systems

4. Gonsalvez, R.A., 1982, Optical Engineering 21(5), pp. 829-832

5. Gonte, F., Araujo, C., Bourtembourg, R., Brast, R., Derie, F., Duhoux, P., Dupuy, C., Frank, C., Karban, R., Mazzoleni, R., Noethe, L., Surdej, I., Yaitskova, N., Wilhelm, R., Luong, B., Pinna, E., Chueca, S., Vigan, A., 2008, Proceedings of the SPIE, Volume 7012, pp. 70120Z-70120Z-12

6. Gonte, F., Yaitskova, N., Derie, F., Araujo, C., Brast, R., Delabre, B., Dierickx, P., Dupuy, C., Frank, C., Guisard, S., Karban, R., Noethe, L., Sedghi, B., Surdej, I., Wilhelm, R., Reyes, M., Esposito, S., Langlois, M., 2006, Proceedings of the SPIE, Volume 6267, pp. 626730

7. Gonte, F., Araujo, C., Bourtembourg, R., Brast, R., Derie, F., Duhoux, P., Dupuy, C., Frank, C., Karban, R., Mazzoleni, R., Noethe, L., Sedghi, B., Surdej, I., Yaitskova, N., Luong, B., Chueca, S., Reyes, M., Esposito, S., Pinna, E., Puglisi, A., Quiros Pacheco, F., Dohlen, K., Vigan, A., 2009, The Messenger, vol. 136, p. 25-31

8. Mocoeur, I., Mugnier, L.M., Cassaing, F., 2007, 21ième Colloque sur le Traitement du Signal et des Images Proceedings of GRETSI 2007

9. Ragazzoni, R., 1996, Journ. Mod. Opt., 43, pp. 289-293

10. Rodrigues, G., Roose, S., Stockman, Y., Gebhardt, S., Schoenecker, A., Villon, P., Preumont, A., 2009, Optical Engineering, Volume 48, Issue 3, pp. 034001-034001-7

11. Roddier, F., 1988, Appl. Opt. 27, 1223-1225

12. Roose, S., Bolsée, J.C., Lecat, J.H., Loix, N., 2006, Sixth International Conference on Space Optics, Proceedings of ESA/CNES ICSO 2006, ESA SP-621. European Space Agency, p.11.1

13. Shack, V., Platt, B.C., 1971, J. Opt. Soc. Am. 61, 656-660

14. Shi, F., Chanan, G., Ohara, C., Troy, M., Redding, D. C., 2004, Appl. Opt. 43, 4474-4481

15. Wilhelm, R., Luong, B., Courteville, A., Estival, S., Gonté, F., Schuhler, N., 2008, Applied Optics, Vol. 47, Issue 29, pp. 5473-5491

16. Yaitskova, N., Dohlen, K., Dierickx, P., Montoya, L., 2005, J. Opt. Soc. Am. A 22, 1093-1105

17. Yaitskova, N., Gonte, F., Derie, F., Noethe, L., Surdej, I., Karban, R., Dohlen, K., Langlois, M., Esposito, S., Pinna, E., Reyes, M., Montoya, L., Terrett, D., 2006, Proceedings of the SPIE, Volume 6267, pp. $62672 Z$ 